\title{
INTERACTIVE IMAGE GEOLOCALIZATION IN AN IMMERSIVE WEB APPLICATION
}

\author{
Mouna Harrach, Alexandre Devaux, Mathieu Brédif \\ Univ. Paris-Est, LASTIG GEOVIS, IGN, ENSG, F-94160 Saint-Mandé, France \\ firstname.lastname@ign.fr
}

Commission II

KEY WORDS: 3D web application, historical images, pose estimation, georeferencing

\begin{abstract}
For a long time people have been interested in the past and history and how we can go back in time using a time machine. And while we cannot invent a time machine, at least not yet, we can create a virtual one. Our "virtual" time machine is an interactive web application that allows users to browse through and navigate within historical images (aerial/terrestrial photographs or postcards) that are projected on a 3D photogrammetric model (point cloud or 3D mesh), thus going back in time and interacting with historical 3D models and images. This was achieved by adopting a semiautomatic approach where the user identifies first 6 to 8 hints on the historical image and the photogrammetric model, then this information is used as an entry data to a photogrammetric software that computes the pose and orientation of the image. The purpose of this work, which is part of the ALEGORIA project, is to preserve cultural heritage, to give the users the opportunity to go back in time and study history of a place or simply discover how their hometown looked some years ago.
\end{abstract}

\section{INTRODUCTION}

This work is part of the ALEGORIA project that aims at facilitating the promotion of iconographic institutional funds collections describing the French territory in various periods going from the interwar period to our days. The collections in sight are of variable sizes, between thousands and hundred thousand elements, and are constituted by iconographic representations, which are most of the time oblique, vertical or terrestrial photographs of the environment. Contrary to the well anchored exploitation which is made of the satellite imagery where the professional practices are many (researchers, institutions and local authorities) and the data well identified and indexed, the promotion of those collections remains confidential and scattered. They are spread within various institutions, digitized partially, generally not or little documented and weakly georeferenced. Nevertheless they represent a rich heritage, little known by the general public and exploited in a way forced by their main users (researchers, institutions and local authorities), in direct consultation at the library or by means of online classic libraries. The development of such a heritage would benefit from tools allowing to automate their collection, to be capable of crossing them and then of studying them better. Their implementation within applications implies to facilitate their apprehension by users not specialists, it supposes to supply friendly tools for the visual exploration of these collections. Therefore to answer these assessments, our work provides an engine for the immersive display of these contents, allowing spatiotemporal navigation and interaction in the $3 \mathrm{D}$ environment enriched with ancient photographic collections.

\footnotetext{
*Corresponding author
}

The proposed approach used in our work, is that for each new image with unavailable or inaccurate georeferencing, the user will navigate immersively in the collection of georeferenced data (DTM, lidar, Aerial images...) to an approximate viewpoint possibly aided by preliminary inaccurate pose estimates. Then the user will be able to give interactive hints to refine the pose and calibration of the new image (scaling, perspective, lens distortion, point correspondences with other 2D image points or points from a 3D virtual globe...). The proposed method is interactive and allows to report and propagate the uncertainty of its estimates. We will present first a short state-of-the-art on the problematic of spatial resection, then we will explain in more details the method we used and its implementation and finally the tests with their results.

\section{BACKGROUND}

The mathematical problematic behind our study is the spatial organization of historical photographs on a cloud of points or a 3D photogrammetric model resulting from a modeling phase. The concept of spatial resection can be a starting point for the development of a spatial referencing system of photographs within 3D architectural scenes (Busayarat, 2008). The term space resection is the name given to the process in which the spatial position and orientation of photograph is determined based on photogrammetric measurements of the images of ground control points appearing on the photograph (Moffitt and Mikhail, 1980). In essence, the space resection makes use of image coordinates and heavily weighted or fixed object space coordinates to determine the positional and rotational elements of a photograph, or of a camera. Following this basic definition, space resection of a single photo can be extended to include the interior orientation parameters, or it can be reduced to include positional elements only. Therefore, the space resection may have 3 parameters $\left(X_{c}, Y_{c}, Z_{c}\right), 6$ parameters $\left(X_{c}, Y_{c}, Z_{c}, \omega, \phi, \kappa\right)$, or more. Since spatial resection is a non linear problem, existing methods involve linearizing the colinearity condition and using an iterative process to determine the final solution through the least squares method (Easa, 2013). The process also requires approximated initial values of the unknown parameters, some of which must be estimated by another least squares solution.

From the existing applications that already tackle this subject while using a different procedure, we mention Smapshot (Blanc et al., 2018), a Swiss web-based participatory virtual globe where users participate in georeferencing historical images of the landscape provided either by the Swiss ETH library or SwissTopo: the Swiss Federal Office of Topography, by clicking a minimum of six well identifiable correspondence points between the image and a 3D virtual globe. The users must have a basic understand- 


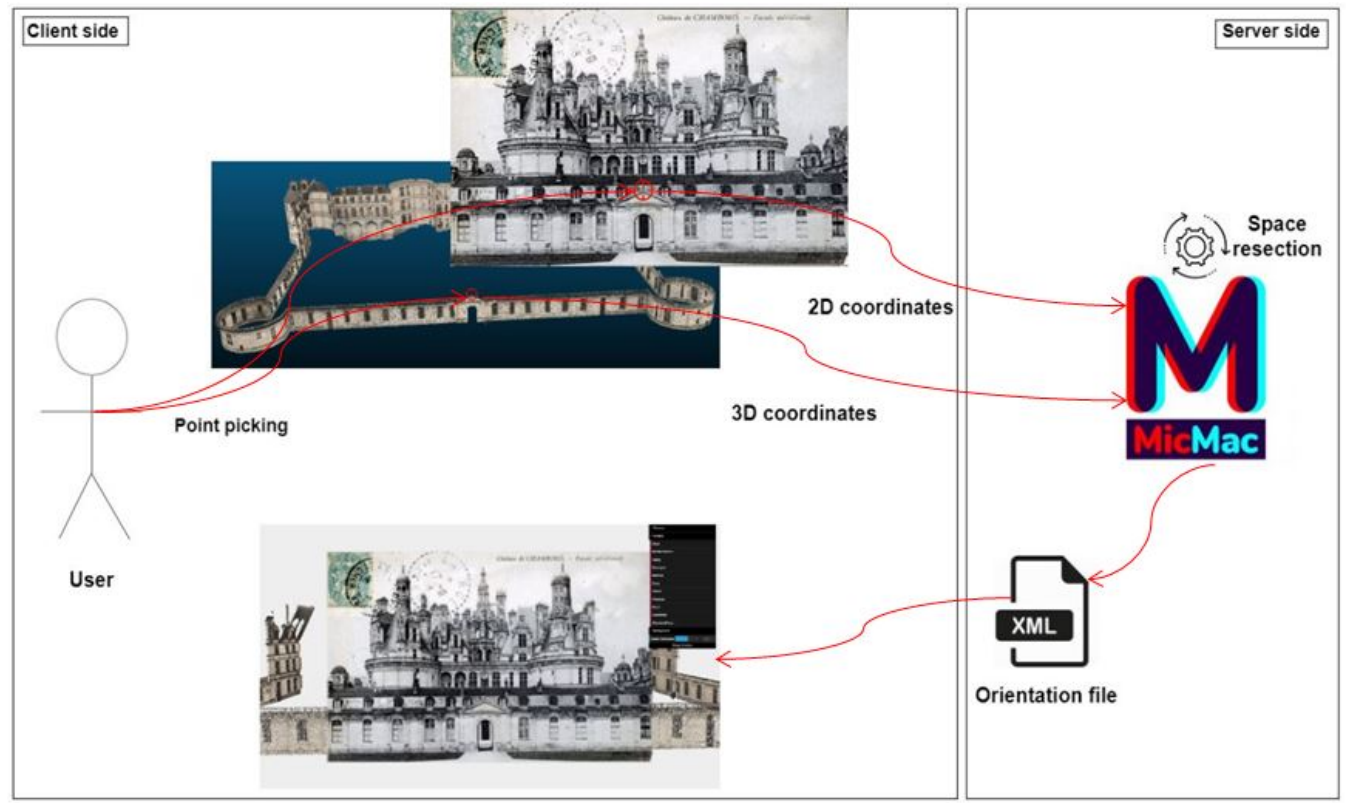

Figure 1. Client side of the application where the user interacts with the web interface and its connection with the server side where computations take place.

ing of the geographic notions as they should first specify the geographical position of the photography on the map, then define the shooting direction, and lastly identify 6 common points between the photography and the virtual globe. Smapshot is limited to the Switzerland territory only, and it relies on the Image Archive team to validate the image after the user have successfully localized the image, which can take up to three working days. This can be very time consuming and meaningless if the image is rejected afterwards.

\section{IMPLEMENTATION}

In order to solve the space resection problematic, we developed a semi-automatic approach to help photogrammetric tools estimate the pose of historical images. Since it is a semi-automatic web-based tool, the user will help georeference the historical photograph by pointing at least 6 to 8 details commonly shared by the photograph and the $3 \mathrm{D}$ model, then the rest of the procedure is completely automatic and provides interactive feedback (Figure. 1). To implement this semi-automatic and interactive online georeferencing process, we relied on our locally developed technology MicMac, a free open-source photogrammetric software for 3D reconstruction (Rupnik et al., 2017) that was exposed as a web-service. MicMac is accessible via command line and provides a large number of commands that can each perform different photogrammetric computations depending on the user's needs. In our context, we mainly used the command Aspro. This tool allows to orientate a set of images with known internal calibration from existing 3D points and their corresponding image projection. After retrieving the pose estimated by the MicMac web-service as an orientation XML file that is parsed into a WebGL camera, we are able to superimpose the historical image on the 3D model. At the first stage, the user interaction (creating point correspondences) and the visualization of the poses estimated by the web-service in the background were both achieved within a simple 3D WebGL application. This application was then incorporated into a platform called iTowns. iTowns is a web framework written in Javascript/WebGL for viewing and exploiting all types of 3D geographic data across the web (Devaux and
Brédif, 2016) therefore it enables the immersive navigation in both recent and historical images.

\section{TESTS AND RESULTS}

The input data used for testing the application was composed of 2 different datasets each providing a different result. The first dataset was a historical photograph accompanied by a 3D point cloud, while in the second dataset we used a DTM (Digital Terrain Model) as the 3D photogrammetric model. For the first dataset, we used a historical postcard of the Chambord Castle in France (Figure. 2a) that we succeeded in georeferencing using a set of 6 control points acquired by pointing certain details (such as intersections, center of objects, corners...) on the postcard and their correspondents on the point cloud (Figure. 2b).

It is very important for the points to be well distributed and to appear on different levels which allowed us to precisely project the historical image on the photogrammetric model.

For the second dataset, we studied two different cases. The first one was a case where the data represented an urban area, while the second one showed a mountain area. In the first case, we retrieved 2D coordinates from a historical aerial photography representing the place of Concorde in Paris, France (Figure. 3a) along with their corresponding 3D coordinates derived from a DTM (Digital Terrain Model) through the iTowns platform (Figure. 3c), we then projected approximately the historical photograph on the DTM as shown in (Figure. 3e).

If we overlook the point of view of the camera, we notice a slight shift between the photograph and the DTM highlighted in red (Figure. 3e), while in the second case where we used the same method but a different photograph of the Mont Blanc (Figure. 3b), we obtain a better result approximation wise as we don't notice any shift this time (Figure. 3f). 


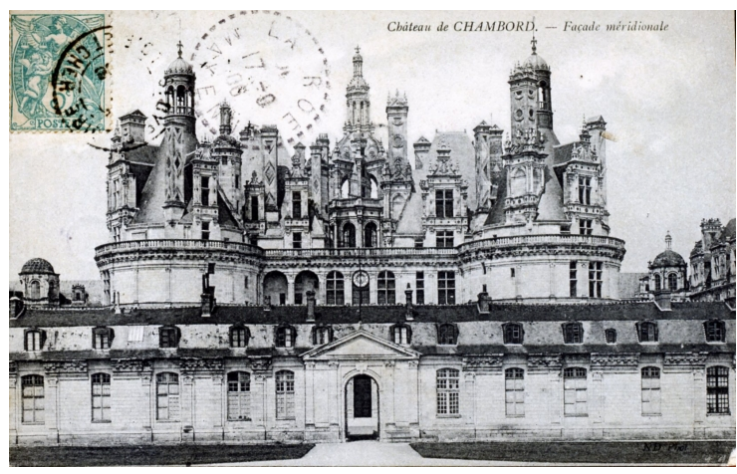

(a) Historical post card

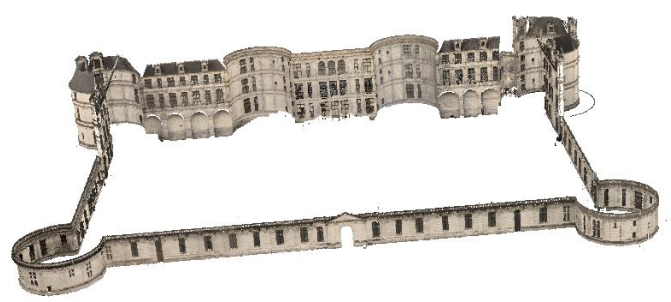

(b) 3D photogrammetric point cloud

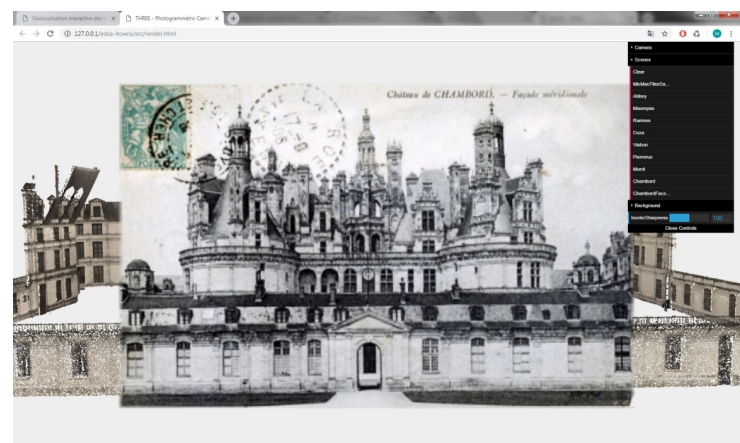

(c) Historical post card re-projected on the 3D point cloud model

Figure 2. Results on the Chambord castle, France.

\section{CONCLUSION}

If we compare the results obtained using the different datasets we can conclude that in the case of an accurate 3D reference model, we recover accurate $3 \mathrm{D}$ coordinates $(\mathrm{X}, \mathrm{Y}, \mathrm{Z})$ therefore the space resection is well achieved, in other words we succeed in projecting the historical image on the photogrammetric model accurately. On the other hand, in the case of approximated input coordinates or coordinates that appear to be in the same plane that leads to inaccuracy in the initial parameters used to solve the space resection equations, therefore the orientation computed does not converge to an acceptable solution.

In order to overcome these issues, we are considering different approaches. The first one is using a mathematical approach for specific cases such as when the control points are all located on the same plane in order to resolve the space resection. The second one is similar to the re-photography (Bae et al., 2010) principle, meaning that instead of having a 2D-3D correspondence, we will have only $2 \mathrm{D}$ coordinates to rely on in solving the space resection problematic.

The main goal of this work is to geolocalize different types of historical images semi-automatically, and it will be especially useful in the future for georeferencing street-level images and even historical movies with a fixed camera.

\section{ACKNOWLEDGEMENTS}

This work is supported by ANR, the French National Research Agency, within the ALEGORIA project, under Grant ANR-17CE38-0014-01.

\section{REFERENCES}

Bae, S., Agarwala, A. and Durand, F., 2010. Computational rephotography. ACM Transactions on Graphics 29(3), pp. Article No.24.

Blanc, N., Produit, T. and Ingensand, J., 2018. A semi-automatic tool to georeference historical landscape images. PeerJ Preprints 6, pp. e27204v1.

Busayarat, C., 2008. Conception d'un système de référencement spatial de photographie de relevé. MIAjournal 0, pp. 1-6.

Devaux, A. and Brédif, M., 2016. Realtime projective multitexturing of pointclouds and meshes for a realistic street-view web navigation. In: Proceedings of the 21 st International Conference on Web3D Technology, Web3D '16, ACM, New York, NY, USA, pp. 105-108.

Easa, S. M., 2013. Space resection in photogrammetry using collinearity condition without linearisation. Survey Review 42(315), pp. 40-49.

El-Ashmawy, K. L. A., 2018. Using direct linear transformation (dlt) method for aerial photogrammetry applications. Geodesy and Cartography 44(3), pp. 71-79.

Moffitt, F. H. and Mikhail, E. M., 1980. Photogrammetry. Harper [and] Row, New York.

Rupnik, E., Daakir, M. and Pierrot Deseilligny, M., 2017. Micmac - a free, open-source solution for photogrammetry. Open Geospatial Data, Software and Standards 2(1), pp. 14. 
The International Archives of the Photogrammetry, Remote Sensing and Spatial Information Sciences, Volume XLII-2/W9, 2019 8th Intl. Workshop 3D-ARCH “3D Virtual Reconstruction and Visualization of Complex Architectures", 6-8 February 2019, Bergamo, Italy

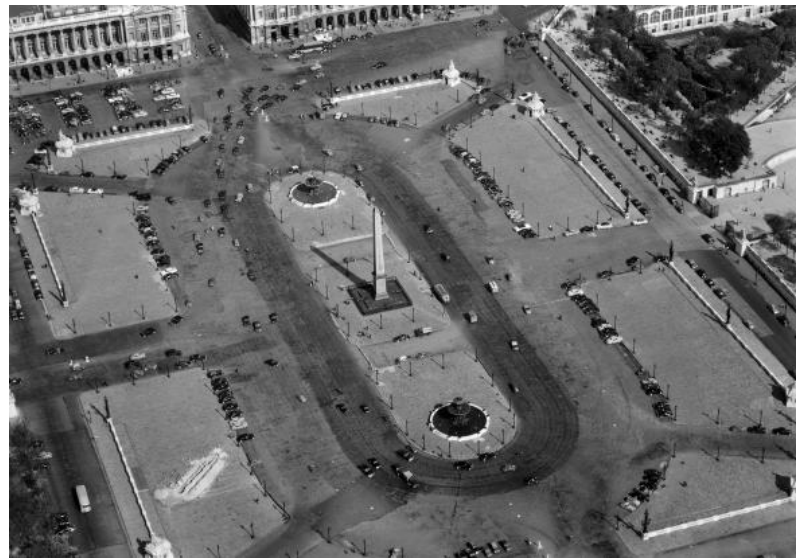

(a) Historical photograph

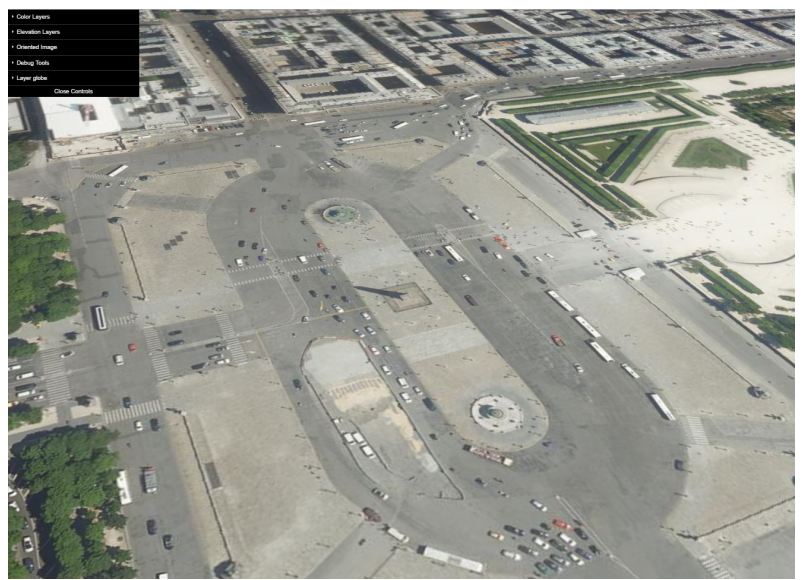

(c) Current DTM textured with orthophoto, viewed in 3D (iTowns)

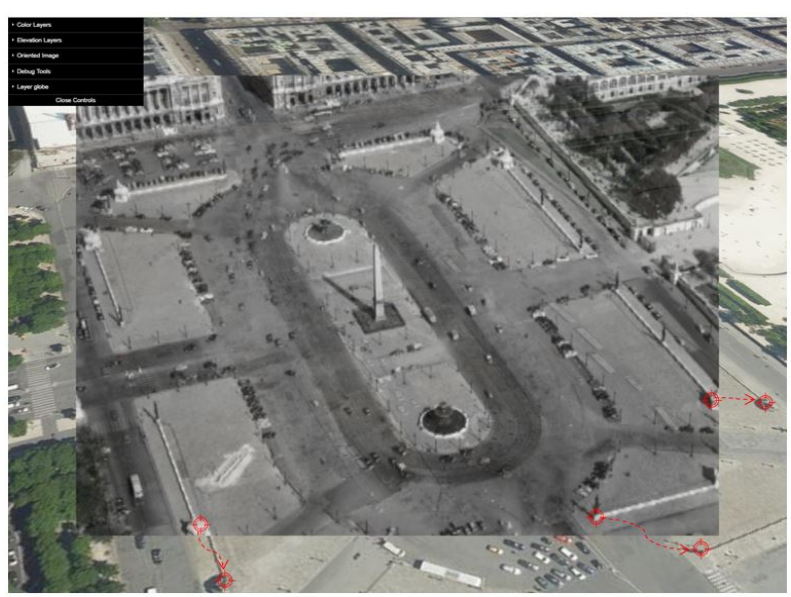

(e) Historical photograph of the place Concorde projected on the DTM in iTowns with the shift highlighted in red.

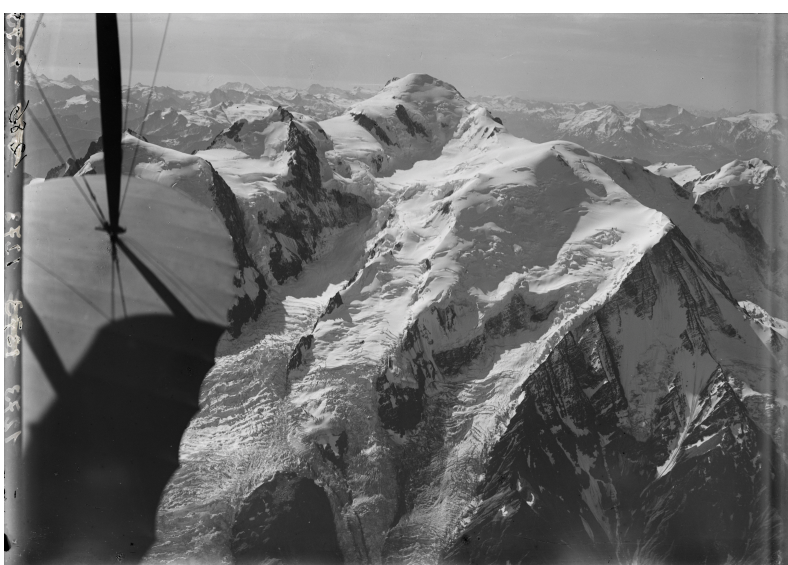

(b) Historical photograph

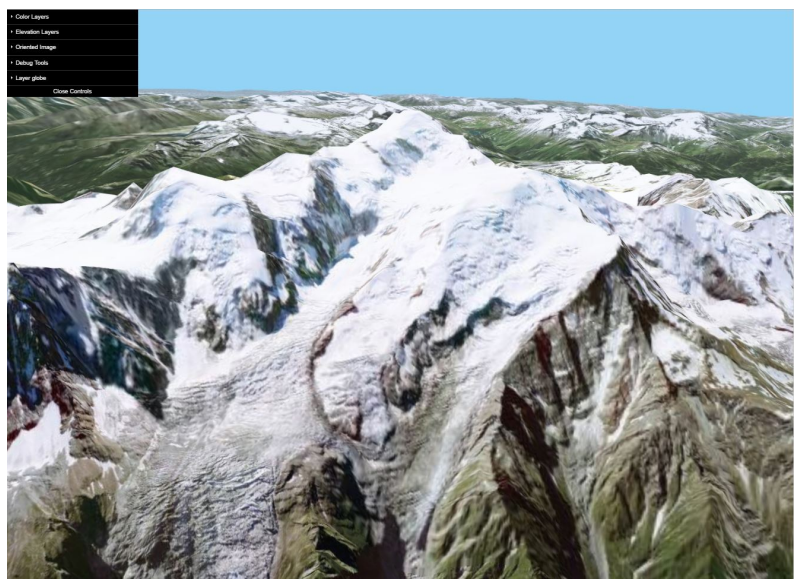

(d) Current DTM textured with orthophoto, viewed in 3D (iTowns)

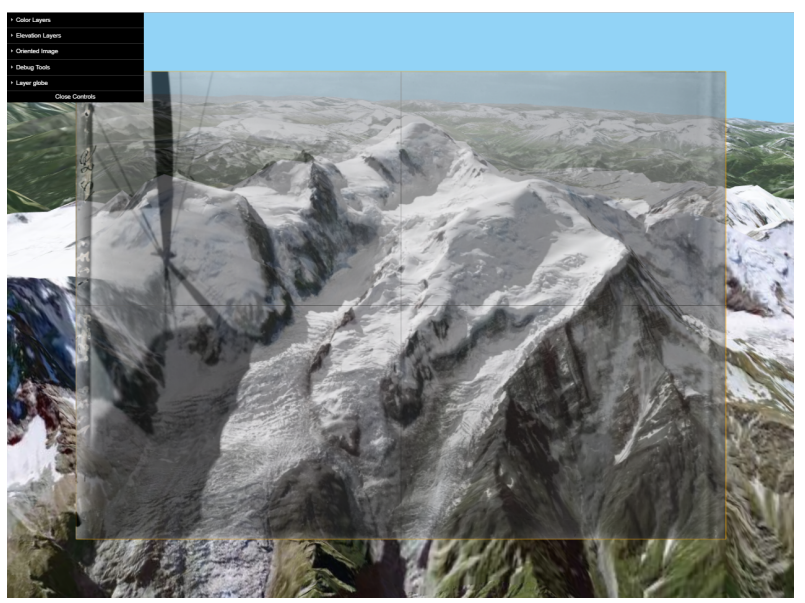

(f) Historical photograph of the Mont Blanc projected on the DTM in iTowns.

Figure 3. Results on the place Concorde in Paris, France (left) and on the Mont Blanc mountain, France (right). 